\title{
Characterization of a multicore fiber image guide for nonlinear endoscopic imaging using two-photon fluorescence and second-harmonic generation
}

\author{
Anikitos Garofalakis \\ Sergei G. Kruglik \\ Tigran Mansuryan \\ André Gillibert \\ Luc Thiberville \\ Frédéric Louradour \\ Christine Vever-Bizet \\ Genevieve Bourg-Heckly
}




\title{
Characterization of a multicore fiber image guide for nonlinear endoscopic imaging using two-photon fluorescence and second-harmonic generation
}

\author{
Anikitos Garofalakis, ${ }^{\mathrm{a}, *, \dagger}$ Sergei G. Kruglik, ${ }^{\mathrm{a}}$ Tigran Mansuryan, ${ }^{\mathrm{b}}$ André Gillibert, ${ }^{\mathrm{c}}$ Luc Thiberville, ${ }^{\mathrm{d}}$ \\ Frédéric Louradour, ${ }^{b}$ Christine Vever-Bizet, ${ }^{a}$ and Genevieve Bourg-Heckly ${ }^{\mathrm{a}}$ \\ a Sorbonne Université, Centre National de la Recherche Scientifique, Institut de Biologie Paris-Seine, Laboratoire Jean Perrin, Paris, France \\ bUniversité de Limoges, XLIM, CNRS UMR 7252, Limoges, France \\ ${ }^{\mathrm{c}}$ Rouen University Hospital, Department of Biostatistics, Rouen, France \\ ${ }^{d} \mathrm{CHU}$ Rouen, Service de Pneumologie, Oncologie Thoracique et Soins Intensifs Respiratoires, Rouen, France
}

\begin{abstract}
Multiphoton microscopy (MPM) has the capacity to record second-harmonic generation (SHG) and endogenous two-photon excitation fluorescence (2PEF) signals emitted from biological tissues. The development of fiber-based miniaturized endomicroscopes delivering pulses in the femtosecond range will allow the transfer of MPM to clinical endoscopy. We present real-time SHG and 2PEF ex vivo images using an endomicroscope, which totally complies with clinical endoscopy regulations. This system is based on the proximal scanning of a commercial multicore image guide (IG). For understanding the inhomogeneities of the recorded images, we quantitatively characterize the IG at the single-core level during nonlinear excitation. The obtained results suggest that these inhomogeneities originate from the variable core geometries that, therefore, exhibit variable nonlinear and dispersive properties. Finally, we propose a method based on modulation of dispersion precompensation to address the image inhomogeneity issue and, as a proof of concept, we demonstrate its capability to improve the nonlinear image quality. () The Authors. Published by SPIE under a Creative Commons Attribution 4.0 Unported License. Distribution or reproduction of this work in whole or in part requires full attribution of the original publication, including its DOI. [DOI: 10.1117/1.JBO.24.10.106004]
\end{abstract}

Keywords: fiber optics; endoscopic imaging; fluorescence microscopy; nonlinear microscopy; medical and biological imaging.

Paper 190020RR received Jan. 20, 2019; accepted for publication Jul. 25, 2019; published online Oct. 23, 2019.

\section{Introduction}

Multiphoton microscopy (MPM) is now a well-established method for tissue structural and functional imaging. ${ }^{1-4}$ Studies have been reported in a variety of tissues, from animal models to human samples, and the technique is also used in vivo for dermatological applications. ${ }^{5-7}$

MPM is of particular value for imaging extracellular matrix (ECM) architecture in label-free tissues since the combination of second-harmonic generation (SHG) and endogenous twophoton excitation fluorescence (2PEF) allows the simultaneous visualization of collagen and elastin, respectively, the main ECM proteins. Several major lung pathologies such as chronic bronchial inflammations, chronic obstructive pulmonary disease, fibrosis, asthma, emphysema, and cancer are characterized by early modifications of the ECM fibrillary collagen and elastin network. A number of investigations on ex vivo animal ${ }^{8-11}$ and human lung samples ${ }^{12-18}$ already demonstrated the potential of the method to assess the pathological changes occurring in ECM organization.

To date, fiber-optic, single-photon confocal autofluorescence endomicroscopy allows in vivo microscopic real-time imaging of the human respiratory tract, during bronchoscopy, from proximal bronchi down to the alveoli. In previous studies, ${ }^{19,20}$ the authors showed that, under the 488-nm single-photon excitation mode, the technique images the elastin network of the proximal and distal bronchial tree but does not give access to

*Address all correspondence to Anikitos Garofalakis, E-mail: agarofalakis@ biospacelab.com

${ }^{\dagger}$ Present Address: BiospaceLab, Nesles-la-Vallée, France the collagen component of the ECM. Translation of MPM to endoscopy potentially offers one way to overcome this limitation: imaging both collagen (through SHG) and elastin (through 2PEF) networks would provide the capability to follow structural remodeling of the ECM in vivo during an endoscopic procedure, which would be of great clinical value.

There have been significant research efforts ${ }^{21,22}$ to transfer table-top MPM to clinical endoscopy by developing miniaturized endomicroscopes using optical fibers both to deliver light to the tissue and to collect the backward-emitted multiphoton tissular signals. Two endomicroscopy techniques have been developed, based either on the distal scanning of a single fiber or on the proximal scanning of a fiber bundle. In the first case, the scanning mechanism is placed at the tip of a single fiber, whereas, in the second case, the scanning mechanism, placed at the fiber entrance, sweeps the laser beam across a multicore image guide (IG), ensuring a sequential illumination of the fiber cores. In recent years, research has been focused mainly on the development of endomicroscopes based on the distal scanning of different types of single fibers. ${ }^{23-30}$ These studies have proven the potential of providing uniform images, but they do not fully comply with the U.S. Food and Drug Administration (FDA) and European Commission (CE) conformity regulations required for a clinical use, namely because of the ability to accurately sterilize this kind of miniaturized device has not been demonstrated yet.

On the other hand, fiber-bundle-based endomicroscopes present a number of advantages: as the scanning mechanism and its electrical control are not included in the probe to be incorporated in the operating channel, there is no restriction on scanner size; the probe is compact, mechanically flexible, and robust, and a real-time acquisition rate $(\sim 10 \mathrm{~Hz})$ can be achieved. 
Moreover, it can be chemically sterilized against bacteria and viruses, in contrast to distal scanning endomicroscopes, and it allows efficient signal collection using a large surface, i.e., the total bundle area. All these advantageous features increase the clinical applicability of the technique. On the other hand, a limitation of this approach is the limited lateral resolution of multifiber IG due to several micrometers core-to-core distance and optical cross talk between the adjacent fiber cores.

Several studies have assessed the potential of multicore fiberbundle-based systems for $2 \mathrm{PEF}$ and/or SHG imaging. Using a 140 -cm-long fiber bundle connected distally to a gradient-index lens and proximally to a pair of diffraction gratings to precompensate the group velocity dispersion occurring in the fiberbundle cores, images from fluorescein-labeled blood vessels in anesthetized rats have been obtained. ${ }^{31}$ The $2 \mathrm{PEF}$ images from fresh cresyl-violet-stained tissue canine bladder were also reported $^{32}$ using a 50-cm-long fiber bundle, without compensating for dispersion and nonlinear effects in the fibers. Some of the present authors developed several systems allowing the precompensation of both linear and nonlinear distortions arising in long single-mode fibers (SMF), ${ }^{33,34}$ and using a specially designed multicore fiber bundle from Fujikura, 2PEF images of human colon cells stained with Rhodamine B were obtained. ${ }^{33}$ Recently, a system employing a fiber bundle has been developed enabling the simultaneous imaging of coherent antiStokes Raman scattering, SHG, and 2PEF in a human dermis sample. ${ }^{35,36}$ The bundle, used only to conduct the excitation wavelengths, is an IG consisting of 10,000 light-guiding cores with a relatively small length of $1 \mathrm{~m}$, and thus, no compensation for dispersion in the fibers was implemented.

However, to the best of our knowledge, no attempt has been reported to perform joint imaging of a tissue by SHG and endogenous (label-free) 2PEF using a fiber-bundle-based endomicroscope directly operable in a routine endoscopic procedure. This means that the instrument should provide real-time imaging to the practitioner and avoid motion artifacts induced by heartbeat and breathing; the fibered probe should be flexible, small-sized, and long enough to be easily inserted in the operating channel of an endoscope, guided along curved lumen of hollow organs and put into contact with the tissue. Moreover, the probe should be fully biocompatible, resistant to decontamination processes and eligible for both $510(\mathrm{k})$ clearance from the U.S. FDA and the European CE conformity marking. All these features can be found today in single-photon endomicroscopes, but, to our knowledge, these requirements are not met by any multiphoton endomicroscope in development.

The aim of this study is to evaluate the possibility of developing a multiphoton fiber-bundle-based endomicroscope, meeting all the requirements for clinical use, and thus immediately transferable into endoscopy at the patient bedside. This endomicroscope would be intended primarily for imaging ECM of elastin and collagen networks. For this purpose, we built an endomicroscope based on a commercial fiber bundle and present the acquired endogenous $2 \mathrm{PEF}$ and SHG images from ex vivo tissue samples. The main focus has been put into quantitative characterization of the properties of this fiber bundle as a nonlinear IG.

\section{Materials and Methods}

\subsection{Multicore Fiber Image Guide}

The IG used in our study is the Gastroflex ${ }^{\mathrm{TM}}$ UHD confocal miniprobe ${ }^{\mathrm{TM}}$ commercialized by Mauna Kea Technologies for use with the single-photon fluorescence endomicroscope Cellvizio ${ }^{\circledR}$. This miniprobe is based on a Fujikura multicore fiber-optic bundle of the FIGH series. The bundle is composed of 30,000 separate optical cores, each one having a diameter no greater than $2 \mu \mathrm{m}$, with center-to-center intercore distance of about $3.5 \mu \mathrm{m}$. The effective working field of view of this miniprobe has a diameter of $600 \mu \mathrm{m}$ and its total length is $3 \mathrm{~m}$. Therefore, it can be used in an operating room, regardless the tissular target to be reached. The outer diameter of the IG is $2 \mathrm{~mm}$, which makes it compatible with any endoscope-operating channel having a minimal diameter of $2.8 \mathrm{~mm}$. The distal end of the bundle is connected to a proprietary micro-objective (MO) with working distance of $60 \mu \mathrm{m}$ and magnification of 2.48, resulting in a lateral resolution of $1.4 \mu \mathrm{m}$ and an axial resolution (optical section thickness) of $9 \mu \mathrm{m}$ (specifications at $488 \mathrm{~nm}$ ).

\subsection{Tissue Sample Preparation}

The human lung samples used in this investigation were prepared by Rouen University Hospital and were obtained from one patient undergoing surgery for lung cancer. The specimens were removed from a lobectomy sample in a healthy zone, remote from the cancer, and fixed in 4\% paraformaldehyde. After fixation, samples were cut into $\sim 1$-mm-thick tissue slices. Image acquisitions were performed on a healthy peripheral alveolar territory sample. Each tissue block was placed on a microscope slide.

Rat tail tendons were dissected from thawed rat tails and stored in phosphate-buffered saline solution (to remove hemoglobin traces) for several minutes before image acquisitions. A part of the tendon was placed on a microscope slide.

\subsection{Experimental Setups}

Two variations of the experimental setup were built: one for 2PEF and SHG imaging of tissue using fiber-bundle IG, and another one for quantitative characterization of the IG at the single-core level.

The setup is shown in Fig. 1. A tunable (690 to $1040 \mathrm{~nm}$ ) Ti: sapphire laser (L) having 80-MHz repetition rate and 70-fs pulse duration (Spectra-Physics Mai Tai/DeepSee) is used as the excitation light source. A half-wavelength plate $\left(\mathrm{WP}_{1}\right.$, Thorlabs AHWP05M-980) together with the Faraday isolator (FI, Newport ISO-05-800-BB-G) are placed at the laser output to vary the pulse energy and also to prevent the parasitic feedback by reflected light. Then, via a pair of reflecting mirrors $\left(\mathrm{M}_{1}\right.$ and $\mathrm{M}_{2}$, Thorlabs PF10-03-P01), the light enters a home-built pulseshaping device, ${ }^{34,37}$ which permits simultaneous second-order dispersion (SOD), third-order dispersion (TOD) dispersion, and nonlinear effect precompensation. This device was initially developed for delivering ultrashort pulses through a long SMF for various endoscopic applications. ${ }^{34,37-40}$ It consists of (i) a short SMF (Thorlabs PM780-HP) with its focusing optics $\left(\mathrm{L}_{1}\right.$ and $\mathrm{L}_{2}$, Thorlabs A260TM-B) and polarization rotation management (WP $\mathrm{W}_{2}$, Thorlabs AHWP05M-980), (ii) mirrors for light delivery $\left(\mathrm{M}_{3}\right.$ to $\mathrm{M}_{6}$, Thorlabs PF10-03-P01), and (iii) a grism-based pulse stretcher $\left(G_{1}\right.$ and $\left.G_{2}\right)$ consisting of a pair of reflection diffraction gratings in close contact with two prisms.

The principle of operation of this precompensation system has been previously described in detail. ${ }^{33,34}$ Briefly, a short piece of SMF introduces spectral and temporal pulse broadening under the combined effects of self-phase modulation and positive (i.e., normal) group-velocity dispersion, effectively decreasing the light intensity entering the first grism $\left(G_{1}\right)$. 
(a)

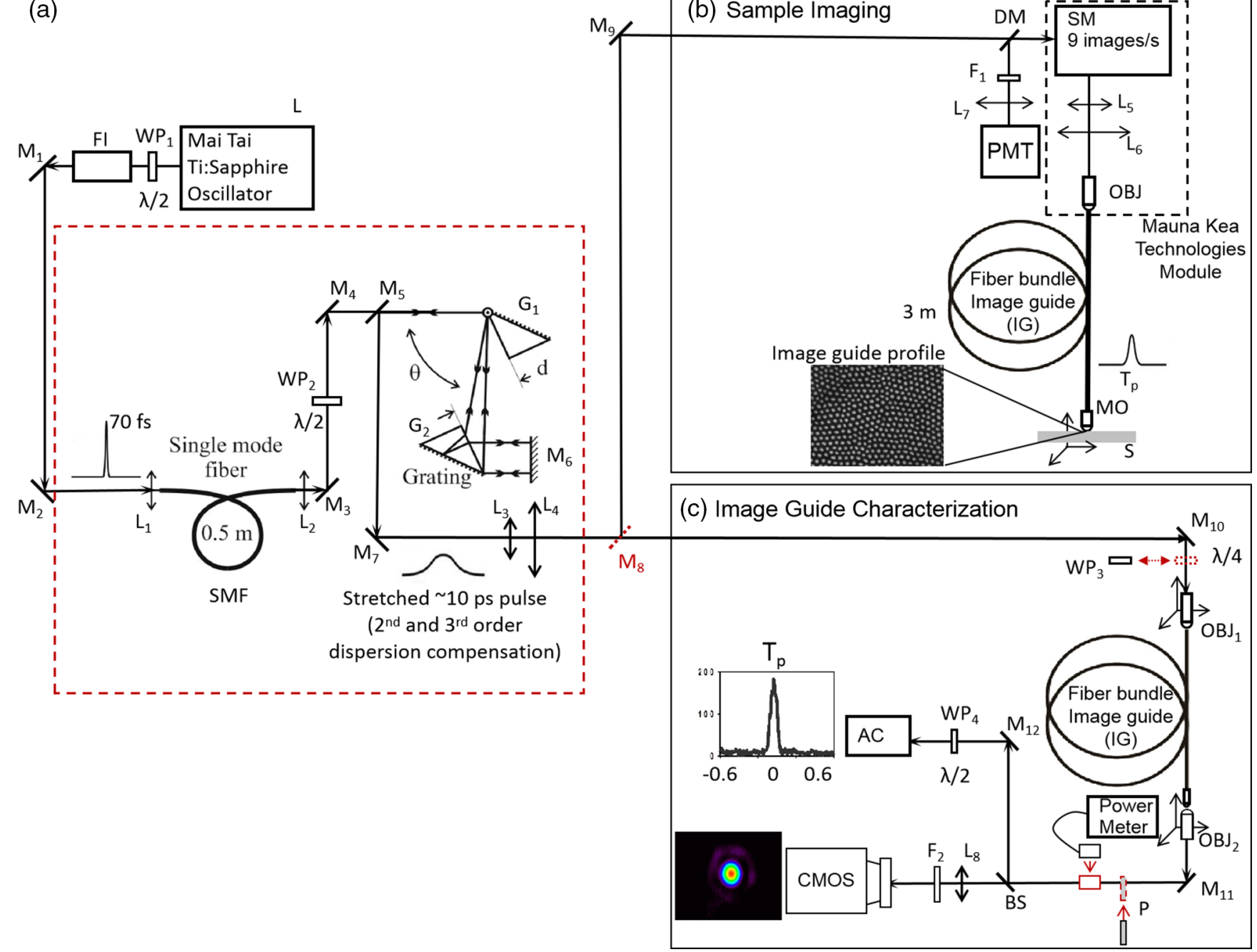

Fig. 1 Experimental setup employing an endoscope-compatible multicore IG for ex vivo 2PEF and SHG imaging of tissular samples and characterization of the same IG at the single-core level. (a) The part of the setup that is common for the two type of measurements; $L$, a femtosecond laser; $W P_{1}$ and $W P_{2}$, halfwaveplates; $F I$, Faraday isolator; $M_{1}$ to $M_{7}$, mirrors; $L_{1}$ to $L_{4}$, lenses; SMF, single-mode fiber; and $G_{1}$ and $\mathrm{G}_{2}$, pair of grisms. (b) Module dedicated to $2 \mathrm{PEF}$ and $\mathrm{SHG}$ imaging; DM, dichroic mirror; $\mathrm{F}_{1}$, dichroic short-pass or bandpass filter; SM, set of two scanning mirrors; OBJ, objective; IG, multicore image guide; and $\mathrm{MO}$, micro-objective lens. The tissue sample, S, is placed on a 3-D stage. The dashed line frame depicts the components composing the pulse-shaping device. (c) Module dedicated to the IG characterization at the single-core level; $\mathrm{WP}_{3}$, quarter-waveplate; IG, multicore image guide; $\mathrm{OBJ}_{1}$ and $\mathrm{OBJ}_{2}$, objectives on 3-D stages; $P$, polarizer; PM, power meter; $B S$, beam splitter; $L_{8}$, lens; $F_{2}$, neutral density filter; CMOS, video camera; $\mathrm{WP}_{4}$, half-waveplate; $A C$, autocorrelator; and $\mathrm{M}_{10}$ to $\mathrm{M}_{12}$, mirrors.

Then, an anomalous dispersive stretcher consisting of two grisms $\left(G_{1}\right.$ and $\left.G_{2}\right)$ produces, in two passes, largely negatively chirped pulses. As a result, after propagation inside the long delivery fiber with positive dispersion and nonlinearity, the pulse impinging on the tissue can be optimally compressed to a value close to or even shorter than the initial laser pulse duration. This is achieved by proper adjustment of the anomalous stretcher, i.e., by changing the distance $d$ between the two grisms and the angle of incidence $\theta$, one can control the desired values of SOD and TOD, respectively. Although a pulse duration of about 10 picosecond (ps) is measured at the stretcher's exit (between mirrors $M_{5}$ and $M_{7}$ ), a value in the range 50 to $100 \mathrm{fs}$ can be routinely obtained after passing through a singlemode 3-m-long fiber. Note that our grisms-based precompensation device is tunable from 790 to $900 \mathrm{~nm} .{ }^{39}$ An excitation wavelength of $850 \mathrm{~nm}$, optimal for suppressing the 2PEF signal from collagen, ${ }^{39}$ was used in this study.

After the pulse-shaping device, the beam passes via an optical delivery system consisting of a mirror $\left(\mathrm{M}_{7}\right.$, Thorlabs PF10-03$\mathrm{P} 01)$ and two lenses $\left(\mathrm{L}_{3}\right.$, Thorlabs AC254-050-B and $\mathrm{L}_{4}$, Thorlabs AC254-100-B) that act as a telescope for expanding the laser beam and adjusting its divergence. Then, depending on the type of experiment, the beam follows different trajectories.

\subsubsection{Module for ex vivo two-photon excitation fluorescence and second-harmonic generation imaging of tissular samples}

For the 2PEF and SHG imaging of tissue, the $\mathrm{M}_{8}$ and $\mathrm{M}_{9}$ mirrors guide the beam to the module presented in Fig. 1(b). 
The collimated light passes through a dichroic mirror (DM, Semrock FF665-Di02-25 × 36) toward a proprietary scanning module (Mauna Kea CellVizio ${ }^{\circledR}$ ) composed of two scanning mirrors (SMs), a telescope $\left(\mathrm{L}_{5}\right.$ and $\left.\mathrm{L}_{6}\right)$, and a coupling objective $(\mathrm{OBJ})$. The laser beam is swept across the proximal end of the multicore IG (Gastroflex ${ }^{\mathrm{TM}}$ UHD miniprobe ${ }^{\mathrm{TM}}$ ) ensuring sequential illumination of all fiber cores. Horizontal line scanning is performed using a $4-\mathrm{kHz}$ resonance mirror while a galvanometric mirror performs frame scanning. The dwell time of the laser light per core is $<1 \mu \mathrm{sec}$ and varies depending on the area scanned. The minimal frame rate of $9 \mathrm{~Hz}$ is set for compatibility with in vivo in-clinic endoscopic application, which requires real-time imaging during intervention on patients. After propagating through the IG, the beam is focused onto the sample tissue (S) by a proprietary MO, which is an integral part of the employed IG. The sample is placed on a three-dimensional (3-D) differential micrometer translation stage; the horizontal in-plane movement is used to manually select the sample's region of interest, whereas the vertical movement enables probing of different tissue depths.

The total average power on the sample is deliberately limited to $40 \mathrm{~mW}$, corresponding to a pulse energy of $0.5 \mathrm{~nJ}$. The $2 \mathrm{PEF}$ and SHG signals are collected by the whole IG in epi-detection configuration, delivered back to the proximal end of the IG, descanned by SMs (as for usual linear fluorescence imaging but without pinhole filtering), reflected by the dichroic beamsplitter (DM) and focused on a fast photomultiplier (PMT, Hamamatsu H10723-110MOD) by a lens $\left(\mathrm{L}_{7}\right)$ with $f=50 \mathrm{~mm}$. Dichroic filter is placed before the PMT for spectral selection of the informative signal: a low-pass filter (Semrock FF01-720/SP25) allows simultaneous recording of $2 \mathrm{PEF}$ and SHG signals, whereas a bandpass filter centered at $427 \mathrm{~nm}$ with 10-nm FWHM (Semrock FF01-427/10-25) allows acquisition of the SHG signal exclusively. The PMT signal is then synchronously processed using a 14-bit digitizer, and two-dimensional images are reconstructed using a proprietary software provided by Mauna Kea Technologies.

The recorded videos are then subjected to postprocessing treatment frame by frame. First, a $2 \times 2$ binning is applied for reducing the read noise. Then a circular averaging filter with a radius of $7.5 \mu \mathrm{m}$ is applied for smoothing the image.

\subsubsection{Module for image guide characterization at single-core level}

For the quantitative characterization of the IG at the single-core level, the $\mathrm{M}_{8}$ mirror is removed and the beam exiting the telescope $\left(\mathrm{L}_{3}\right.$ and $\left.\mathrm{L}_{4}\right)$ is focused onto the proximal end of the fiberbundle IG by a $20 \times$ objective $\left(\mathrm{OBJ}_{1}\right.$, Nikon Plan, NA 0.4$)$. The proximal end of the fiber bundle is positioned on a 3-D steppermotor translation stage with a minimal incremental motion of $50 \mathrm{~nm}$ and unidirectional repeatability of $100 \mathrm{~nm}$ (three linear stages M-111.12S and stepper motor controller C-600, Physik Instrumente). The choice of an arbitrary single core is performed by moving the stage along the $X-Y$ plane. Then, translation along the optical $(Z)$ axis is used to optimize the coupling into this core. Various cores can be selected by moving the 3-D stage in the $X-Y$ plane without changing the $Z$ axis position. Light exiting the distal end of the IG is collimated by a $50 \times$ objective $\left(\mathrm{OBJ}_{2}\right.$, Olympus MS Plan, NA 0.80) and then split by a beam sampler (BS, Thorlabs BSF10-B) into two measurement channels containing relevant optical elements: a half-waveplate $\left(\mathrm{WP}_{4}\right.$, Thorlabs AHWP05M-980) before an autocorrelator
(AC), a lens, $\mathrm{L}_{8}$, and a neutral density filter $\left(\mathrm{F}_{2}\right.$ Thorlabs NE10A-B) before a CMOS camera. Both $X-Y$ and $Z$ positions of the stepper motor are fine-tuned to optimize the signal of the cores on the CMOS camera. The process of this optimization consists in looking at the spatial distribution of one core on the CMOS camera. Then the stepper motor moves to maximize the core intensity and minimize the cross talk of the targeted core with respect to the adjacent ones. Optionally, a polarizer ( $\mathrm{P}$, Thorlabs LPNIR050) can be placed in front of the mirror, $\mathrm{M}_{11}$, and a quarter-waveplate ( $\mathrm{WP}_{3}$, Thorlabs AQWP05M-980) before the $\mathrm{OBJ}_{1}$.

The above setup was used for measuring the following parameters.

a. Average power of transmitted light with a power meter (PM, Newport 1918C with the 918D-SLOD3R measuring head) from which the pulse energy was calculated. The power was usually limited to $10 \mathrm{~mW}$, corresponding to pulse energy of $125 \mathrm{pJ}$.

b. Pulse duration, by AC (APE PulseCheck, long-pulse version: 50 fs to $50 \mathrm{ps}$ ). Signal is maximized by rotating the half-waveplate $\mathrm{WP}_{4}$.

c. The degree of polarization (defined as $\mathrm{Pol}=$ $[I \max -I \min ] /[I \max +I \min ])$, by inserting and rotating the polarizer $\mathrm{P}$ in front of the PM. For measurements with circularly polarized input light, the quarter-waveplate $\left(\mathrm{WP}_{3}\right)$ was placed before the first objective, $\mathrm{OBJ}_{1}$.

d. Beam spatial profile, by CMOS camera (Thorlabs DCC1545M). The lens $\mathrm{L}_{8}$ with $f=50 \mathrm{~cm}$ is used for focusing the parallel beam into the camera, providing real-time images of the cores with a field of view of $25 \times 25 \mu \mathrm{m}^{2}$. The neutral density filter $F_{2}$ prevents signal saturation on the camera.

\subsection{Statistics}

Spearman's correlation coefficients were computed to assess the correlations of pulse energy and degree of polarization with pulse duration. The $p$-value and $95 \%$ confidence intervals (CIs) were estimated by bias-corrected accelerated $\left(\mathrm{BC}_{a}\right)$ bootstrap according to Efron's method. ${ }^{41}$ A correlation coefficient was considered negligible if it was below 0.30 , weak if it was between 0.31 and 0.70 , strong if it was between 0.71 and 0.90 , and very strong if it was above 0.91 . Correlation coefficients were compared to the value 0 (null hypothesis). A $p$-value below 0.05 was considered statistically significant. A semilogarithmic scale was used for pulse duration in scatter plots since the pulse duration distribution was highly skewed. All statistics were computed in R statistical software (version 3.5.0, The R Foundation for Statistical Computing, Vienna, Austria).

\section{Results and Discussion}

\subsection{Unlabeled Tissues Imaging}

Two types of dissected samples were imaged with the first configuration of the setup; (a) $2 \mathrm{PEF}$ of human alveolar territory sample without any staining, and (b) SHG from a rat tail tendon (Fig. 2).

These images were recorded in selected regions of interest with the size $190 \times 140 \mu \mathrm{m}$ (see also Videos 1 and 2). 
Elastin, which is found in abundance in human alveoli, is considered as the main source of $2 \mathrm{PEF}$ signal; the image in Fig. 2(a) provides information on the structure of elastin network. In Fig. 2(b), SHG signal originates from tendons rich in collagen.

Then, we sought to explore the possibility of parallel assessment of 2PEF and SHG signals within the same region of a sample (Fig. 3). A dissected portion of a rat tail was continuously imaged and manually scanned until a tendon structure appeared in the SHG spectral window [see Fig. 3(a)]. Then without moving the sample, a low-pass filter was positioned (see Sec. 2.3.1), and the SHG filter was removed in front of the PMT. From the recorded image containing both $2 \mathrm{PEF}$ and SHG signals (data not shown), we performed a pixel-to-pixel subtraction of the image shown in Fig. 3(a) to obtain the signal of 2PEF [Fig. 3(b)].

Our approach aimed to be conformed with the medical regulations related to standard endoscopic procedures, meaning that the fiber guide had to be approved for clinical endoscopic imaging. Moreover, real-time imaging is indispensable to endoscopic practices. The above results presented in Figs. 2 and 3 are, to the best of our knowledge, the first images of $2 \mathrm{PEF}$ of elastin and SHG of collagen from ex vivo tissue without any external labeling, using a multicore IG and at endoscopy-relevant frame rate. The images shown here were subjected to postprocessing by applying a combination of numerical filters, as described above. However, the time of image treatment was significantly shorter than the time interval between two successive frame acquisitions at $9 \mathrm{~Hz}$. Therefore, such a treatment can be
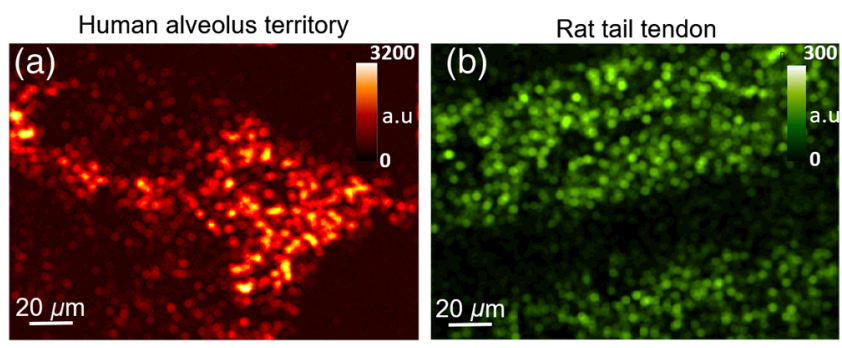

Fig. 2 The 2PEF and SHG ex vivo real-time imaging with the use of endoscopic multicore IG. (a) Still image of the 2PEF signal taken from a cine loop of $9 \mathrm{~Hz}$ of a human alveolus territory from a biopsy sample (see Video 1, MP4, 8.88 MB [URL: https://doi.org/10.1117/ 1.JBO.24.10.106004.1]). (b) Still image taken from a cine loop of $9 \mathrm{~Hz}$ showing the SHG signal of the tendons of rat tail excised sample (see Video 2, MP4, 4.58 MB [URL: https://doi.org/10.1117/ 1.JBO.24.10.106004.2]). Additional 2PEF experiments using fluorescein-labeled tissue were performed and the signals obtained were stronger (see Video 3, MP4, 1.00 MB [URL: https://doi.org/10.1117/ 1.JBO.24.10.106004.3]). directly integrated into real-time monitoring of $2 \mathrm{PEF}$ and SHG signals during in-clinic endoscopy intervention.

Although these first results are promising, the recorded images are far from being optimal. First, the signals, especially in the case of SHG, were relatively weak and, second, the images exhibited high spatial inhomogeneity contrary to images provided by distal imaging schemes.

Regarding signal intensity, a number of factors contribute to the observed problem. First, we chose an endoscopically relevant fast frame rate of $9 \mathrm{~Hz}$ which translates in a very short exposure time of laser excitation per fiber in one frame. As a result the non-linear signals from nonlabeled tissue are weak. We performed additional $2 \mathrm{PEF}$ experiments using fluorescein-labeled tissue and we obtained much stronger signals with better quality of images (Video 3). On the other side, the total average power of femtosecond radiation at the distal end of an IG was limited at a certain level $(40 \mathrm{~mW})$ and cannot be substantially increased to avoid tissue damage. Importantly, in this study, we used the available scanning module from Mauna Kea Technologies, the optics of which were optimized for linear excitation in the visible range and not for nonlinear regime using near-infrared (NIR) light at $850 \mathrm{~nm}$. Moreover, we would like to stress that the signal intensity is not the most important parameter per se, as useful information is derived from the morphology of elastin and collagen networks. To accurately resolve the network, a few dozen photons per pixel above the dark current threshold is sufficient. Therefore, we believe that gradual improvement of both scanning and registration modules will allow one to obtain signals sufficiently strong for real-time in-clinic endoscopic imaging of unlabeled tissue.

The second major issue, which is image spatial inhomogeneity, seems to be much more important, and, as described in the following sections, further experiments were performed for the better understanding of the underlying problems.

\subsection{Uniform Sample Imaging}

The postprocessing treatment improves image quality in Figs. 2 and 3; however, the image inhomogeneity is still present. To investigate this problem in detail, experiments were performed on homogeneous samples to examine the signal intensity variation between the cores. The results are shown in Fig. 4 .

Fluorescein water solution $(2.5 \mu \mathrm{M})$ and barium borate $(\alpha$-BBO) crystal were used for obtaining reference images of 2PEF [Fig. 4(a)] and SHG [Fig. 4(b)], respectively. In both cases, the recorded images are surprisingly highly heterogeneous; the corresponding intensity histograms are shown in panels of Figs. 4(c) and 4(d). The core intensities exhibit big variability. In the linear regime with continuous-wave (cw) excitation, the variability in transmission of individual cores
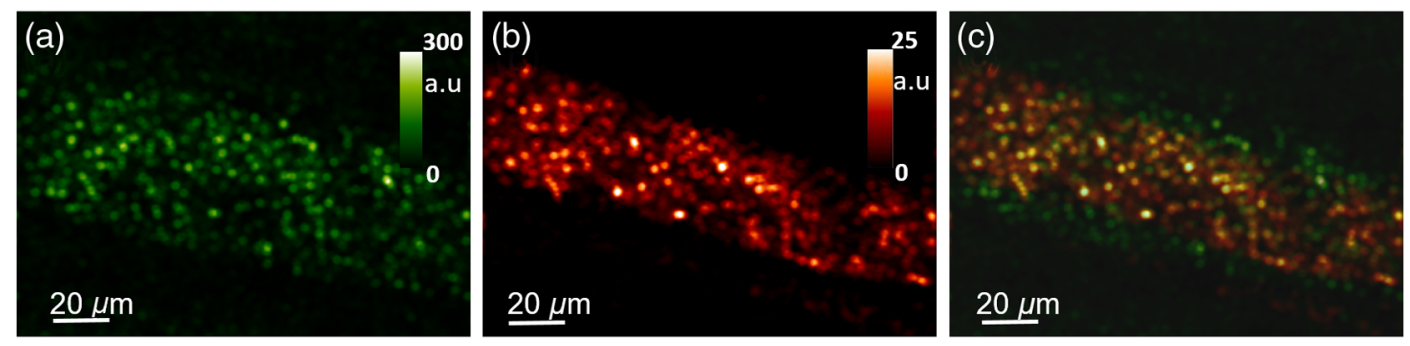

Fig. 3 (a) SHG and (b) 2PEF in-vitro imaging of a tendon from a dissected rat tail sample. The module of Fig. 1(b) has been used for this acquisition. Both images correspond to the same field of view of the sample. (c) Fusion image of (a) and (b). 

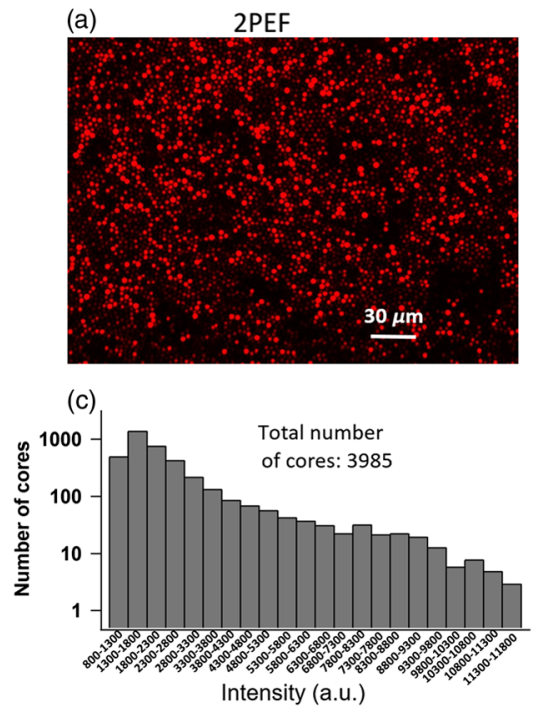

(b)

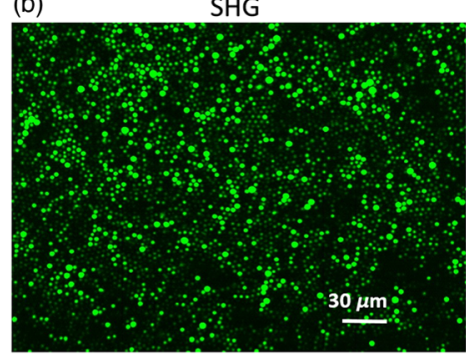

(d)

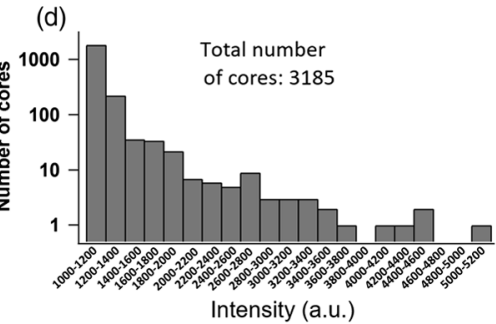

Fig. 4 The distribution of the intensities of the cores of the IG. (a) An image of 2PEF measurement of a fluorescein solution. (b) The corresponding image for the case of a SHG measurement of a BBO crystal. (c), (d) The distributions of the cores based on their intensities following their segmentation as shown in (a) and (b). The range of values for the (a) image was from 811 to 1760 a.u. Similarly, the range of values for the (b) image was from 1030 to 5180 a.u.

also exists. However, it is much lower and, therefore, can be compensated by proper image scaling procedure based on calibration using a fluorophore solution as a standard. However, in the case of nonlinear imaging, an analogous scaling procedure is not immediately possible as it leads to very large correction errors due to very large spread of intensities between the cores.

\subsection{Multicore Fiber Image Guide Characterization at Single-Core Level}

We examined the origin of nonlinear image heterogeneity by studying the IG at the single-core level using the module described in Sec. 2.3.2. In total, 107 different individual cores randomly located within the IG were examined. The distributions of pulse duration, pulse energy, and degree of polarization at the distal exit are presented in Fig. 5. Pulse duration of a substantial part of examined cores lies between 70 and $500 \mathrm{fs}$ $(n=30,28 \%)$, and, within this group, $53 \%$ of cores exhibit pulse durations between 100 and 200 fs [insert in Fig. 5(a)]. However, for the majority of cores $(n=64,60 \%)$, pulse duration is measured to be longer than $1 \mathrm{ps}$ and may even reach 7.4 ps. This means that the precompensation stretcher device $\left(G_{1}\right.$ and $\left.G_{2}\right)$ does not properly compensate the SOD and TOD in the multicore IG for the ensemble of cores but only for some of them. These longer pulse durations translate into almost zero-intensity image points as both $2 \mathrm{PEF}$ and SHG signals depend inversely on the pulse duration.

The pulse energy distribution is shown in Fig. 5(b). The energy of incident pulses was set to $130 \pm 5 \mathrm{pJ}$ at the proximal entrance of the IG. At the distal exit, the pulse energy is measured to be within the range from 10 to $35 \mathrm{pJ}$. About $35 \%$ of the cores $(n=36)$ exhibited output energies between 25 and $30 \mathrm{pJ}$, and the maximum value is measured at $35 \mathrm{pJ}$, giving a coefficient of transmission of about $27 \%$. Note that in this study we do not focus on energy transmission optimization but rather on a robust comparative characterization of different individual cores. Clearly, the pulse energy distribution is rather compact and does not exhibit big variations as compared to those of pulse duration. Finally, when pulse energies are plotted against pulse durations [Fig. 6(a)] of the corresponding cores, the correlation is zero or negligible (rho $=-0.01,95 \% \mathrm{CI}=-0.19$ to +0.16 ).

To study the birefringence due to light propagation in a single core of the IG, we measured the degree of polarization of transmitted pulses and its distribution, as shown in Fig. 5(c). It varies between $3 \%$ and $55 \%$, with $59 \%$ of the cores $(n=63)$ exhibiting a value below $25 \%$. This result confirms that cores are optically anisotropic and alter the linear polarization of an incident light. At the same time, no correlation or a negligible correlation (rho $=+0.07,95 \% \mathrm{CI}=-0.14$ to +0.28 ) between the degree of polarization and pulse duration was found [Fig. 6(b)], suggesting that the induced birefringence is not the main cause of pulse duration variations.

To verify the above conclusion, a modified version of the precedent experiment was performed. The polarization of an incident beam was changed from linear to circular using the quarter-waveplate $\left[\mathrm{WP}_{3}\right.$, Fig. $\left.1(\mathrm{c})\right]$ inserted just before the proximal side of the IG. Durations of the output pulses with and without the quarter-waveplate were measured for 54 individual cores, and the result is shown in Fig. 7. The change from linear to circular polarization of the incident beam does not substantially alter the output pulse durations' distribution, which remains very broad, i.e., from 70 fs to 7.4 ps. This result confirms that the state of polarization during propagation along the fiber core does not influence in a major way the transmitted pulse duration.

Note that the difference between the two distributions of pulse duration for linearly polarized incident light [Fig. 5(a) versus the gray bars of Fig. 7] is due to different individual cores studied in each experiment. One can clearly see that, in spite of substantial local differences, the general tendency remains the same: both distributions are very broad, extending 2 orders of magnitude, from 70 fs to about 7 ps.

Finally, we examined the cross talk between adjacent cores. For this study, CMOS camera images of spatial distribution of transmitted core intensities were used; two representative 

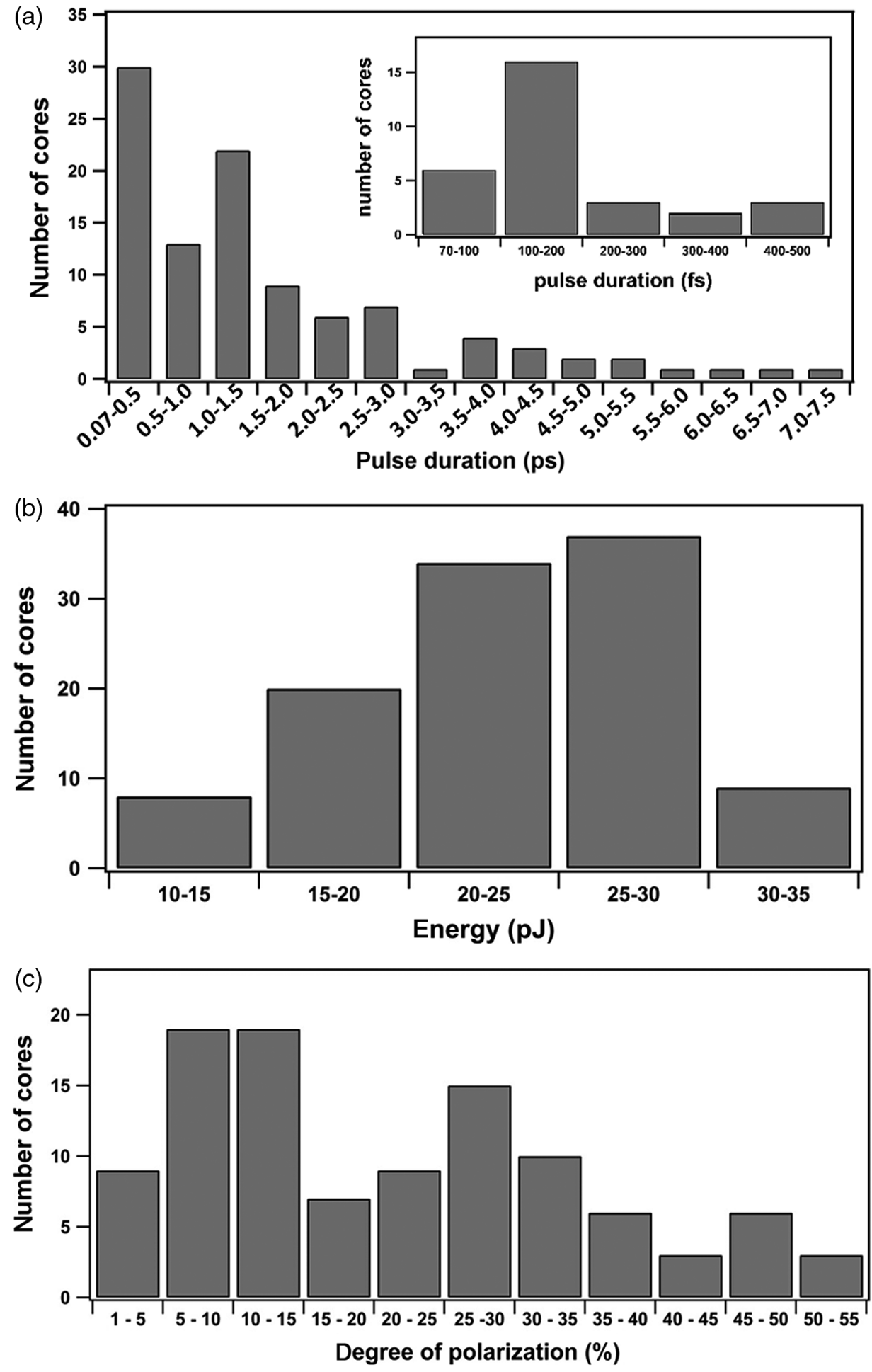

Fig. 5 Histogram plots from parameters measured by the module of the setup of Fig. 1(c) designed for the evaluation of the IG at the single-core level. (a) Distribution of pulse duration, (b) transmitted pulse energy, and (c) degree of polarization over the single cores of multicore IG. The insert in panel (a) shows the extended distribution of pulse duration between 70 and $500 \mathrm{fs}$.

examples are shown in Fig. 8. In both images, the strong peak from the studied core is located in the middle of the camera, whereas adjacent cores with a much lower intensity are also visible around the targeted core. Figure 8(a) shows the particular case of strong cross talk where the intensities from adjacent cores amount up to $44 \%$ of the targeted core peak. However, the latter case is rare and for the large majority of studied cores, the cross talk effect is minimal, with adjacent peak intensities below $10 \%$ of the main peak; a typical example is shown in Fig. 8(b). Intensity profiles along the white lines in Figs. 8(a) and 8(b) are shown in Figs. 8(c) and 8(d), respectively. These data reveal insignificant cross talk between the adjacent cores for Gastroflex $^{\mathrm{TM}}$ UHD confocal miniprobe ${ }^{\mathrm{TM}}$ IG for NIR light at $850 \mathrm{~nm}$. 

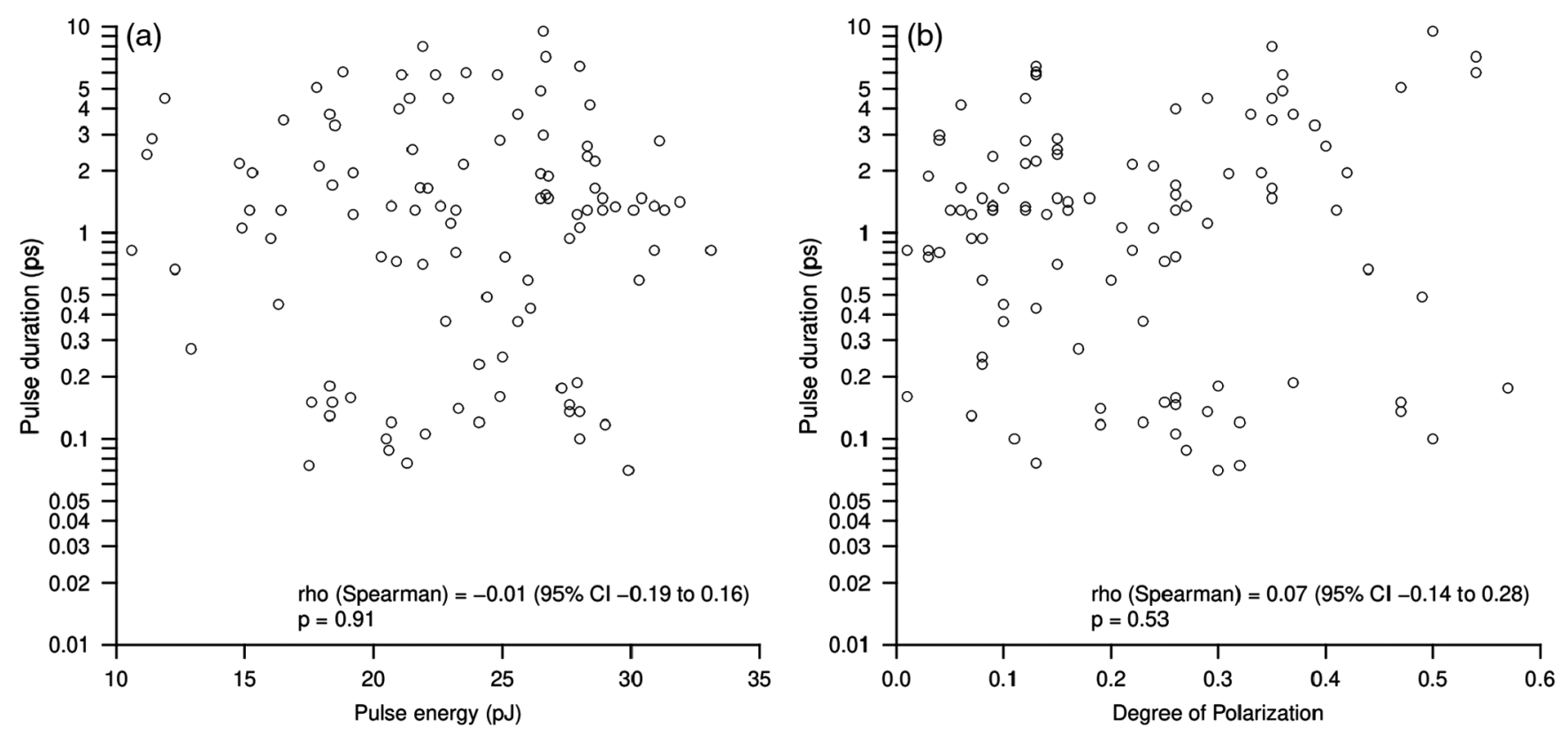

Fig. 6 Scatter plots of (a) pulse energy and (b) degree of polarization against pulse duration. In each case, the Spearman's correlation coefficient (rho) and the $95 \% \mathrm{Cl}$ are given.

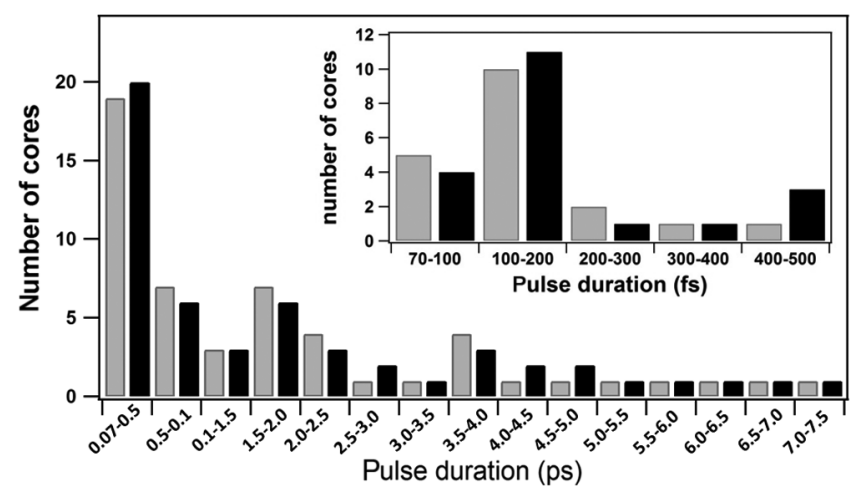

Fig. 7 The effect of circular versus linear input polarization on pulse duration of transmitted light. Gray bars represent distribution of pulse duration in the case of linearly polarized incident light, and black bars represent the case of circular polarized incident light.

There is in fact a trade-off between core uniformity and cross talk; if the transversal core geometries were exactly the same (i.e., cores with identical phase velocities), the cross talk between the adjacent cores would be very strong because of intercore coupling. Fujikura fiber-optic bundle of the FIGH series was specifically designed to reduce the cross talk, thus increasing the resolution of the IG. This is achieved by manufacturing every adjacent core with statistically different transversal profile in size and shape, as depicted in the onset of Fig. 1(b). This intended and well-controlled inhomogeneity is not detrimental for intensity transmission in the linear imaging mode because it can be compensated through image processing. In the case of nonlinear excitation using $2 \mathrm{PEF}$ and SHG with femtosecond pulses, the situation is different. The interaction between the propagating ultrafast light pulse and the core medium depends on the square of intensity and is detrimentally affected by core geometry inhomogeneity.

For a given single core, by fine-tuning the precompensation stretcher, pulse durations in the order of 100 fs can always be achieved at the exit of the core. Indeed, as mentioned in the description of the grism-based stretcher, the value of the introduced anomalous dispersion is adjusted in order to correctly compensate the positive dispersion in the cores of an IG. Modifying the distance between the two grisms $(d)$ and/or the incidence angle $(\theta)$ onto the stretcher [see Fig. 1(a)] permits independent tuning of SOD and TOD to the desired values. The pulses that are anomalously dispersed at the exit of the stretcher can be temporally compressed during their propagation through any chosen core of the bundle.

In the case of our multicore IG, the stretcher should be tuned in a way for precompensating the dispersion of all cores. In our approach, we chose to keep the incidence angle $\theta$ constant and tune the distance $d$ between the grisms [Fig. 1(a)]. The criterion for the optimal distance $d$ was chosen based on mean intensity of the whole IG. However, each core responds individually to the distance $d$, i.e., values of $d$ that maximize the intensity for some cores are not optimal for the others. This observation shows that each core has its own dispersion and nonlinearity. We conclude that optimal precompensation cannot be achieved simultaneously for the ensemble of cores in the bundle.

\subsection{Effect of Modulating the Intergrism Distance on the Nonlinear Image Homogeneity}

Following the above findings, we propose a method for improving the homogeneity of the acquired nonlinear images using a modulation of the dispersion precompensation. This approach is based on the observation that different $d$ values (hence precompensations of SOD) are optimal for different groups of cores. As a proof of concept, an experiment was performed where the distance $d$ was changed in five steps $(\Delta d=0.5 \mathrm{~mm})$ from its initial value. Images of $2 \mathrm{PEF}$ from a fluorescein aqueous solution were recorded at all six precompensation settings. Figure 9(a) shows the whole recorded image at initial $d$ position. A region of interest of $30 \times 20 \mu \mathrm{m}$ was chosen [frame in Fig. 9(a)] and shown in Fig. 9(b) for the six different distances $d$ between the grisms. At each $d$ value, the signal of core intensities varies, as can be seen 


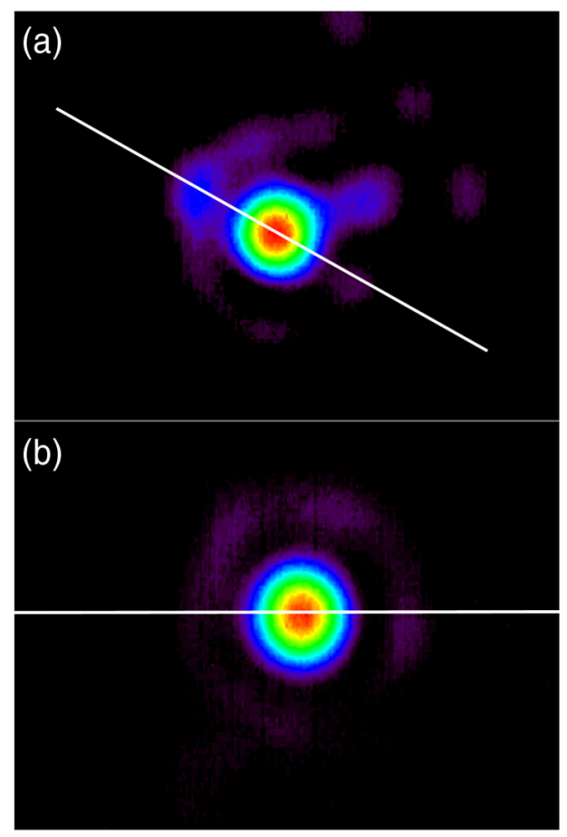

(c)

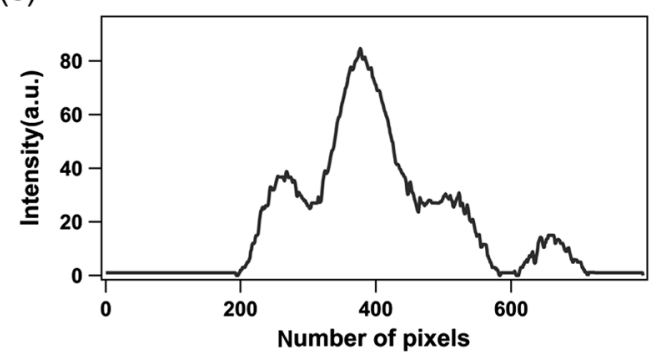

(d)

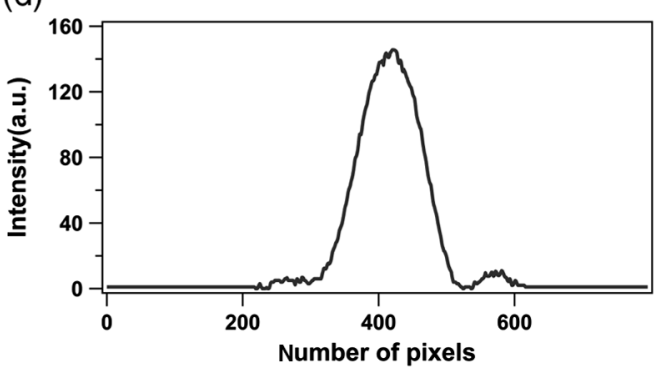

Fig. 8 The cross talk effect of the IG. (a) Image showing a single core of the IG as measured by the CMOS camera of the setup of the Fig. 1(c) as laser light passes through the cores. In this image, a cross talk signal from the adjacent cores is shown. (b) Image showing a single core with typical cross talk signal from the adjacent cores. (c), (d) The distributions of the signal of the cores along the white lines of (a) and (b), respectively.

in Fig. 9(d), for the three chosen cores marked by orange circles in the upper panel of Fig. 9(b). By superposing and averaging the six images of Fig. 9(b) pixel by pixel, a synthetic mean image can be created [Fig. 9(c)], which is more homogeneous than any of the constituent images. Indeed the aggregated image in Fig. 9(c) exhibited less variance in the core intensities $\left(\sigma^{2}=1.3 \times 10^{3}\right)$ than any of the six images in Fig. $9(\mathrm{~b})\left(\sigma^{2}=\right.$ $2.7 \times 10^{3}$ to $\left.5.3 \times 10^{3}\right)$.

\subsection{Future Perspectives for Improving the Image Homogeneity}

We demonstrated that modulation of the dispersion precompensation is a valuable method for improving the image quality. For implementing this method in our setup, an accessible technical solution would consist in modulating the dispersion at high frame rate with a variability amount defined by the results in Fig. 9. In this case, several successive images, having complementary inhomogeneities, would have to be properly associated in order to form a combined final image with optimal homogeneity. One possible discrete dispersion modulation arrangement could be achieved using a relatively simple optomechanical device in combination with the already-existing pulse stretcher. This additional device could be composed of a set of parallel glass plates of different thicknesses positioned in front of a mirror in the image space of a converging lens. A one-dimensional galvanometric mirror, synchronized with the imaging system frame rate and positioned in the focal plane of the lens, could allow quick sequential illumination of various plates, one image frame corresponding to one glass thickness. Due to the above mirror, the double-pass configuration would allow avoiding the transverse displacement of the beam during dispersion modulation.
Other realizations of precompensation modulation, for example, using acousto- or electro-optical beam deflection, are also possible and will be the subject of further studies.

In the experiment depicted in Fig. 9, we note also the existence of cores with very low intensity, regardless of the value $d$. This phenomenon is attributed to nonoptimal coupling of NIR light into the IG since both the scanning module and the IG were initially designed for $\mathrm{cw}$ excitation in the visible range. Indeed, from Fig. 7(b) one can see that, in the conditions of optimal static single-core coupling, the variance of the transmitted energy is rather low; thus, the NIR pulsed laser radiation can in principle be efficiently coupled into any core of the entire IG, provided the optimal matching conditions are satisfied during the proximal beam scanning. Therefore, a new scanning module specifically optimized for NIR laser light and synchronized with an ad hoc precompensation modulation system is to be designed and built to achieve the optimal image quality during real-time in vivo endoscopy.

It is noteworthy that, for an IG with high intrinsic inhomogeneity, the proposed precompensation modulation method leads either to substantial lowering of the useful frame rate or to the reduction of the exposure time in one core. Therefore, in the future, instead of working with a commercially available imaging guide being fabricated and distributed for decades by Fujikura Ltd, one may consider developing a new bundle possessing an optimal equilibrium between cores homogeneity, cores distance, and cross talk between cores that effectively reduces the spatial resolution of the resulting nonlinear image. For such an "optimal" IG, the proposed precompensation modulation procedure is expected to be much faster or eventually may even not be required.

Note that several research groups across the world ${ }^{42,43}$ are now actively working on the development of new imaging multicore bundles with increased resolution and homogeneity. 
(a)

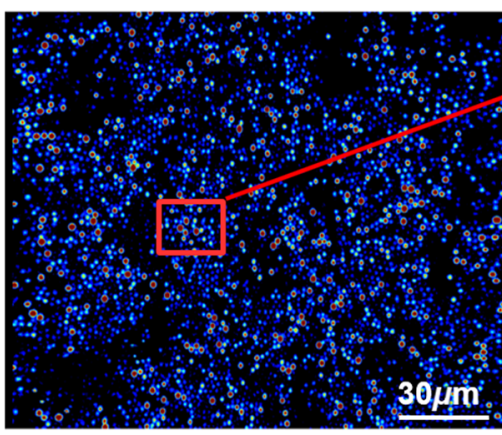

(c)

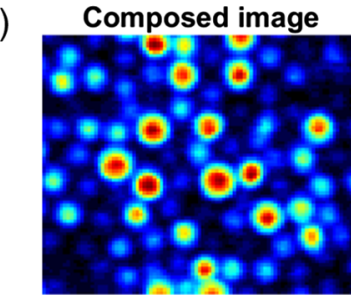

(b)
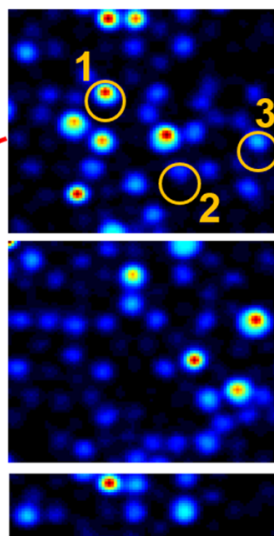

$\Delta d=0.0 \mathrm{~mm}$

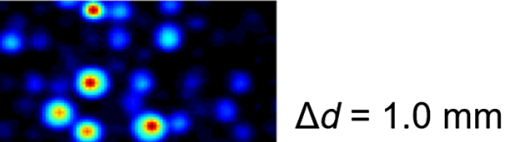

$\Delta d=0.5 \mathrm{~mm}$
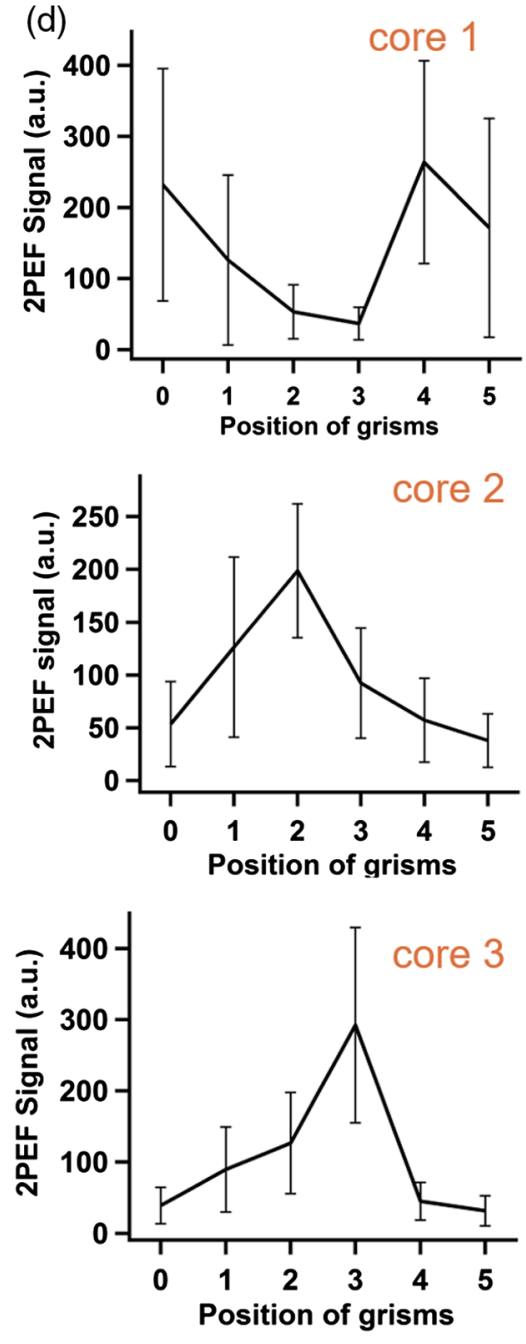

Fig. 9 A method for improving the homogeneity of nonlinear images based on modulation of the distance $d$ between the grisms, hence on modulation of precompensation of SOD. (a) A 2PEF image of a fluorescein solution $(2.5 \mu \mathrm{M})$. (b) Six zoomed images corresponding to six different distances $d$ between the grisms. The parameter $d$ changes in steps of $0.5 \mathrm{~mm}$. (c) Composed image by the mean intensity projection of the six images of (b). (d) The variations of the signals for the three encircled cores of (b). The different points correspond to the different distances between the grisms.

Such IGs should exhibit diameter sizes and mechanical properties that would make them good candidates to be inserted in commercial endomicroscopic probes currently manufactured. Future development of new optical fiber technologies, in particular one based on air-silica microstructures, ${ }^{42}$ represents, in our opinion, one promising route toward nonlinear endomicroscopy and its effective application in the clinic.

\section{Conclusions}

We report a nonlinear endomicroscopy apparatus employing multicore fiber IG compatible with real-time in-clinic endoscopy procedure. The system allowed us to obtain the first real-time cine loops of $2 \mathrm{PEF}$ of elastin and SHG images of collagen from dissected tissues of human alveoli and rat tail tendon, respectively, without any labeling. A frame rate of $9 \mathrm{~Hz}$ was achieved with a total excitation power of $40 \mathrm{~mW}$ at $850 \mathrm{~nm}$. These results suggest good potential for future in-clinic application of nonlinear fiber-bundle-based endomicroscopy, although the obtained image quality is far from optimal, being highly inhomogeneous. To examine this, we performed a rigorous study of the multicore IG employed (Fujikura FIGH series) at a single-core level. We concluded that the nonlinear image inhomogeneity is primarily due to strong variation across the bundle of core transversal geometries, the various cores having variable dispersions. Finally, we proposed a correction method based on modulation of dispersion precompensation to address the image inhomogeneity issue and demonstrated its capability to improve the nonlinear image quality.

\section{Disclosures}

The authors declare that there are no conflicts of interest related to this article.

\section{Acknowledgments}

We are grateful to Mauna Kea Technologies for providing the scanning module and the image acquisition software in the 
frame of a former project funded by the Agence Nationale de la Recherche (ANR).The major part of the experimental equipment used in this study was funded by the ANR (project ONLin vivo ANR-08-TECS-0006-01).

\section{References}

1. M. Strupler et al., "Second harmonic imaging and scoring of collagen in fibrotic tissues," Opt. Express 15(7), 4054-4065 (2007).

2. J. Chen et al., "Multiphoton microscopy study of the morphological and quantity changes of collagen and elastic fiber components in keloid disease," J. Biomed. Opt. 16(5), 051305 (2011).

3. F. Aptel et al., "Multimodal nonlinear imaging of the human cornea," Invest. Ophthalmol. Visual Sci. 51(5), 2459-2465 (2010).

4. J. Lin et al., "Assessment of liver steatosis and fibrosis in rats using integrated coherent anti-Stokes Raman scattering and multiphoton imaging technique," J. Biomed. Opt. 16(11), 116024 (2011).

5. K. König et al., "Clinical two-photon microendoscopy," Microsc. Res. Tech. 70(5), 398-402 (2007).

6. H. G. Breunig et al., "Multiphoton excitation characteristics of cellular fluorophores of human skin in vivo," Opt. Express 18(8), 7857-7871 (2010).

7. K. Koenig, "Hybrid multiphoton multimodal tomography of in vivo human skin," IntraVital 1(1), 11-26 (2012).

8. A.-M. Pena et al., "Three-dimensional investigation and scoring of extracellular matrix remodeling during lung fibrosis using multiphoton microscopy," Microsc. Res. Tech. 70(2), 162-170 (2007).

9. S. Zhuo et al., "Nonlinear optical microscopy of the bronchus," J. Biomed. Opt. 13(5), 054024 (2008).

10. T. Abraham et al., "Minimally invasive multiphoton and harmonic generation imaging of extracellular matrix structures in lung airway and related diseases," Pulm. Pharmacol. Ther. 24(5), 487-496 (2011).

11. I. Pavlova et al., "Multiphoton microscopy and microspectroscopy for diagnostics of inflammatory and neoplastic lung," J. Biomed. Opt. 17(3), 036014 (2012).

12. C.-C. Wang et al., "Differentiation of normal and cancerous lung tissues by multiphoton imaging," J. Biomed. Opt. 14(4), 044034 (2009).

13. T. Abraham and J. Hogg, "Extracellular matrix remodeling of lung alveolar walls in three dimensional space identified using second harmonic generation and multiphoton excitation fluorescence," J. Struct. Biol. 171(2), 189-196 (2010).

14. X. Xu et al., "Multimodal non-linear optical imaging for label-free differentiation of lung cancerous lesions from normal and desmoplastic tissues," Biomed. Opt. Express 4(12), 2855-2868 (2013).

15. K. Tilbury et al., "Second harmonic generation microscopy analysis of extracellular matrix changes in human idiopathic pulmonary fibrosis," J. Biomed. Opt. 19(8), 086014 (2014).

16. G. Tjin et al., "Quantification of collagen I in airway tissues using second harmonic generation," J. Biomed. Opt. 19(3), 036005 (2014).

17. M. Jain et al., "Multiphoton microscopy: a potential 'optical biopsy' tool for real-time evaluation of lung tumors without the need for exogenous contrast agents," Arch. Pathol. Lab. Med. 138(8), 1037-1047 (2014).

18. R. M. Kottmann et al., "Second harmonic generation microscopy reveals altered collagen microstructure in usual interstitial pneumonia versus healthy lung," Respir. Res. 16, 61 (2015).

19. L. Thiberville et al., "In vivo imaging of the bronchial wall microstructure using fibered confocal fluorescence microscopy," Am. J. Respir. Crit. Care Med. 175(1), 22-31 (2007).

20. L. Thiberville et al., "Human in vivo fluorescence microimaging of the alveolar ducts and sacs during bronchoscopy," Eur. Respir. J. 33(5), 974-985 (2009).

21. B. A. Flusberg et al., "Fiber-optic fluorescence imaging," Nat. Methods 2(12), 941-950 (2005).

22. L. Fu and M. Gu, "Fibre-optic nonlinear optical microscopy and endoscopy," J. Microsc. 226(Pt. 3), 195-206 (2007).

23. H. Bao et al., "Fast handheld two-photon fluorescence microendoscope with a 475 microm x 475 microm field of view for in vivo imaging," Opt. Lett. 33(12), 1333-1335 (2008).

24. C. M. Brown et al., "In vivo imaging of unstained tissues using a compact and flexible multiphoton microendoscope," J. Biomed. Opt. 17(4), 040505 (2012).
25. G. Ducourthial et al., "Development of a real-time flexible multiphoton microendoscope for label-free imaging in a live animal," Sci. Rep. 5, 18303 (2015).

26. D. G. Ouzounov et al., "Dual modality endomicroscope with optical zoom capability," Biomed. Opt. Express 4(9), 1494-1503 (2013).

27. A. Lombardini et al., "High-resolution multimodal flexible coherent Raman endoscope," Light Sci. Appl. 7(1), 10 (2018).

28. W. Liang et al., "Nonlinear optical endomicroscopy for label-free functional histology in vivo," Light Sci. Appl. 6(11), e17082 (2017).

29. F. Akhoundi et al., "Compact fiber-based multi-photon endoscope working at $1700 \mathrm{~nm}$, , Biomed. Opt. Express 9(5), 2326-2335 (2018).

30. C. H. Hage et al., "Towards two-photon excited endogenous fluorescence lifetime imaging microendoscopy," Biomed. Opt. Express 9(1), 142-156 (2018).

31. W. Göbel et al., "Miniaturized two-photon microscope based on a flexible coherent fiber bundle and a gradient-index lens objective," Opt. Lett. 29(21), 2521-2523 (2004).

32. K. Thomsen et al., "Development of a confocal and two-photon endomicroscope-preliminary results of qualitative evaluation," Med. Laser Appl. 25(3), 166-172 (2010).

33. M. Lelek et al., "Coherent femtosecond pulse shaping for the optimization of a non-linear micro-endoscope," Opt. Express 15(16), 10154-10162 (2007).

34. C. Lefort et al., "Pulse compression and fiber delivery of 45 fs Fourier transform limited pulses at $830 \mathrm{~nm}$," Opt. Lett. 36(2), 292-294 (2011).

35. A. Lukić et al., "Fiber probe for nonlinear imaging applications," J. Biophotonics 9(1-2), 138-143 (2016).

36. A. Lukic et al., "Endoscopic fiber probe for nonlinear spectroscopic imaging," Optica 4(5), 496-501 (2017).

37. M. Kalashyan et al., "Ultrashort pulse fiber delivery with optimized dispersion control by reflection grisms at $800 \mathrm{~nm}$, , Opt. Express 20(23), 25624-25635 (2012).

38. C. Lefort et al., "Characterization, comparison, and choice of a commercial double-clad fiber for nonlinear endomicroscopy," J. Biomed. Opt. 19(7), 076005 (2014).

39. D. A. Peyrot et al., "Development of a nonlinear fiber-optic spectrometer for human lung tissue exploration," Biomed. Opt. Express 3(5), 840-853 (2012).

40. C. Lefort et al., "Sub-30-fs pulse compression and pulse shaping at the output of a 2-m-long optical fiber in the near-infrared range," J. Opt. Soc. Am. B 31(10), 2317-2324 (2014).

41. B. Efron, "Better bootstrap confidence intervals," J. Am. Stat. Assoc. 82(397), 171-185 (1987).

42. H. A. C. Wood et al., "High-resolution air-clad imaging fibers," Opt. Lett. 43(21), 5311-5314 (2018).

43. B. Morova et al., "Fabrication and characterization of large numerical aperture, high-resolution optical fiber bundles based on high-contrast pairs of soft glasses for fluorescence imaging," Opt. Express 27(7), 9502-9515 (2019).

Anikitos Garofalakis received his $\mathrm{PhD}$ in biomedical physics in 2007 from the University of Crete. Currently, he is the head of the Research and Development (R\&D) Department of the BiospaceLab Company. His work focuses on the development of optical imaging systems for biomedical applications. Prior to joining BiospaceLab, he pursued a career in academic research where he worked on the fusion of various imaging techniques in hybrid multimodal schemes.

Sergei G. Kruglik defended his $\mathrm{PhD}$ thesis in physics and mathematics in 1995 at the Institute of Physics, Minsk, Belarus. Following 15 years of research at this institute, he continued his endeavors at scientific laboratories throughout the world, in Japan, France, The Netherlands, and Canada. $\mathrm{He}$ is now a research engineer at Sorbonne University, Paris, France. His scientific interests are at the crossroads of laser physics, biophotonics, and life sciences, combining advanced laser techniques with optical spectroscopies and medical imaging.

Tigran Mansuryan received his $\mathrm{PhD}$ in laser physics in 2008 from the Physics Department of Yerevan State University. From 2009 to 2014, he was a postdoctoral researcher at XLIM, Limoges University. From 2014 to 2018, he was a R\&D engineer at KAMAX Innovative System. 
$\mathrm{He}$ is now a researcher at XLIM, Limoges University. His research is focused on nonlinear optical methods of microscopy, fiber endoscopy, fiber nonlinearity, ultrafast optics, and femtosecond technologies.

André Gillibert, after a bachelor's degree in pure mathematics, studied medicine at Rouen and Caen universities (2004 to 2017). He holds a master's degree in methodology biostatistics and clinical research (Paris-Sud, 2016). Currently, he is a hospital assistant lecturer in the Department of Biostatistics of Rouen University Hospital.

Luc Thiberville is a professor of respiratory medicine and head of the Respiratory, Thoracic Oncology and Respiratory Care Medicine Department at Rouen University Hospital, France. His research fields include precancerous lesions of the bronchial tree and endoscopic detection and treatment of lung cancer. He introduced and is developing the technique of in vivo endomicroscopy of the respiratory tract, focusing on microimaging of peripheral lung nodules, proximal preneoplastic epithelium, bronchial lung cancers, and interstitial lung disease.

Frédéric Louradour is a professor at XLIM Institute (CNRS 7252), Limoges University, France. His preferred topics are nonlinear optics, femtosecond pulse characterization and shaping, and propagation in optical fibers applied to biomedical instrumentation. He studied endomicroscopy working with polarization or multiphoton or fluorescence lifetime imaging and using an image guide made of a highresolution bundle of thousands of optical fibers or using a single dual-cladding fiber associated to a miniature fiber scanner.

Christine Vever-Bizet has a PhD in biophysics. She joined, in 2004, the Pierre and Marie Curie University, now known as Sorbonne University, in the Jean Perrin Laboratory, CNRS UMR 8237. Her scientific interests focus on photodynamic therapy, cellular and tissular autofluorescence spectroscopy and imaging, as well as confocal laser linear and nonlinear endomicroscopy

Genevieve Bourg-Heckly holds a PhD in physics. After twelve years of professional experience in laser and medical imaging industries, she joined the Pierre and Marie Curie University (currently known as Sorbonne-University, Paris, France) to develop projects in biomedical optics. Her research interests are in the field of noninvasive diagnostic methods based on optical spectroscopy and imaging. Over the past several years, her studies have mainly focused on the development of single-photon and multiphoton endomicroscopies. 